\title{
The definitive management of Hirschsprung's disease with the endorectal pull-through procedure
}

\author{
Theodore Z. Polley, Jr1, 2, Arnold G. Coran", 2, and John R. Wesley ${ }^{1,2}$ \\ ${ }^{1}$ Section of Pediatric Surgery, University of Michigan Medical School \\ 2 Mott Children's Hospital, Ann Arbor, Michigan, USA
}

\begin{abstract}
From 1 July 1974 to 31 August 1985, 99 patients with Hirschsprung's disease were treated at the University of Michigan Mott Children's Hospital; 75 were initially diagnosed and treated at our institution. Of these 75 patients, $55 \mathrm{had}$ standard rectosigmoid disease and 20 had longsegment disease. The endorectal pull-through (ERPT) procedure was used consecutively in 73 of the 75 patients; the other two children died, one because of total intestinal aganglionosis and the other due to severe congenital anomalies, prior to a definitive procedure. Of the 24 patients referred by other institutions, 12 underwent repeat pull-through procedures and 12 required lesser operations to deal with the complications of prior procedures. There were no postoperative deaths and no permanent enterostomies among the 73 consecutive ERPT patients. The follow-up in this series ranges from 4 months to 10 years, and all patients have fecal continence with an average of 3-4 stools daily. Our treatment plan involves the use of suction rectal biopsy for diagnosis and leveling colostomy in the neonatal period. An endorectal pull-through procedure is then carried out between 8 months and 1 year of age. The excellent functional results, $0 \%$ operative mortality, low morbidity, and technical ease of performing the ERPT underscore our enthusiasm for continued use of this procedure in Hirschsprung's disease.
\end{abstract}

Key words: Hirschsprung's disease - Diagnosis Endorectal pull-through

Offprint requests to: Theodore Z. Polley, Jr., Room F7516, Box 066, Mott Children's Hospital, Ann Arbor, MI 48109, USA

\section{Introduction}

Increasing experience with intestinal obstruction in the newborn period and the evaluation of the constipated child continue to indicate that Hirschsprung's disease, or congenital aganglionosis, is an integral part of the differential diagnosis [5]. Since the first description of Hirschsprung's disease by Harold Hirschsprung in 1888, three procedures with their modifications, endorectal pull-through (ERPT), the Duhamel procedure, and the Swenson abdominal-perioneal pullthrough, remain the definitive operations of choice. Throughout our 11-year experience at the University of Michigan Mott Children's Hospital, we have continued to favor the modified endorectal pull-through. Our experience with this procedure in 73 patients represents the focus of this presentation.

\section{Materials and methods}

From 1 July 1974 through 31 August 1985, we had the opportunity to care for 99 patients with Hirschsprung's disease, representing cases primarily diagnosed and treated at our institution as well as those referred from other hospitals. Of these, $35(35 \%)$ presented in the newborn period, i. e., within 30 days of birth. Seventy percent of our patients were male: a $2.3: 1$ male-to-female ratio. Of the 94 patients under 8 years of age, the mean age at presentation was 6.3 months. The other 5 patients were $33,17,16,14$, and 8 years of age at the time of initial diagnosis. The common presenting signs and symptoms were observed in our patients, including bilious vomiting, failure to pass meconium within the first $48 \mathrm{~h}$ of life, bowel obstruction, abdominal distension, and constipation. Two newborns presented with intestinal perforation one with free intraperitoneal air and the other with a localized abscess with sepsis. In addition to Hirschsprung's disease, 15 patients (15\%) had other anomalies, including Down's syndrome in 8 and Smith-Lemli-Opitz syndrome in 1. 
Table 1. Treatment of 99 patients with Hirschsprung's disease at the University of Michigan Mott Children's Hospital 1. july 1974 , to 31. august 1985

\begin{tabular}{|c|c|c|c|}
\hline \multicolumn{2}{|c|}{$\begin{array}{l}73 \text { consecutive endorectal } \\
\text { pull-through (ERPT) procedures }\end{array}$} & \multirow[t]{10}{*}{$\begin{array}{l}24 \text { referred following initial procedure } \\
\text { at outside institution }\end{array}$} & \multirow[t]{10}{*}{$\begin{array}{l}2 \text { with untreated } \\
\text { disease }\end{array}$} \\
\hline & $\mathrm{X}$ & & \\
\hline 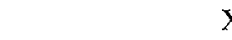 & $\mathrm{X}$ & & \\
\hline $\mathrm{X}$ & $\mathrm{X}$ & & \\
\hline $\mathrm{X}$ & $\mathrm{X}$ & & \\
\hline $\mathrm{X}$ & $\mathrm{X}$ & & \\
\hline $\mathrm{X}$ & $\mathrm{X}$ & & \\
\hline 55 with & 18 with & & \\
\hline standard disease & long-segment & & \\
\hline (rectosigmoid) & disease & & \\
\hline
\end{tabular}

Table 2. Twelve patients referred with complications following a pull-through procedure done at another institution not requiring revision or redo

\begin{tabular}{lll}
\hline Initial procedure & Indications for referral & Treatment \\
\hline Swenson (6 patients) & Rectovesical fistula (1) & Repair (1) \\
& Stricture (1) & Permanent ileostomy (1) \\
& Incontinence and/or constipation (4) & Enema program (4) \\
ERPT (4 patients) & Stricture (1) & Colostomy (1) \\
& Cuff abscess (1) & Drainage and ureterolysis (1) \\
& Enterocolitis (1) & Medical management (1) \\
& Cuff abscess + 180 torsion of pull-through (1) & Colostomy followed by colostomy closure (1) \\
Ileostomy (1 patient) & Severe nutritional depletion (1) & Parenteral nutrition (1) \\
Colostomy (1 patient) & Colostomy stricture (1) & Revision of leveling colostomy (1) \\
\hline
\end{tabular}

Table 3. Summary of 12 patients referred following initial pull-through at another institution requiring revision

\begin{tabular}{lll}
\hline Initial procedure & Indications for referral & Treatment \\
\hline Swenson (5) & Constipation (1) & Myotomy (1) \\
& Stricture (3) & Redo Swenson (3) \\
& Anastomotic disruption (1) & Redo Swenson (1) \\
Duhamel (5) & Constipation (3) & Septectomy (3) \\
& Constipation (1) & Redo Duhamel (1) \\
ERPT (1) & Stricture and abscess (1) & Permanent colostomy (1) \\
Colectomy (1) & Pull-through of aganglionic bowel (1) & ERPT (1) \\
\hline
\end{tabular}

It is most meaningful to divide our patient population into two groups: those originally diagnosed and treated at our hospital (75 patients) and those managed at other institutions and then referred for further care and/or treatment of complications ( 24 patients: see Table 1). The first group included 55 patients with standard rectosigmoid disease. Three-quarters were male, and all underwent modified ERPT. Follow-up in these patients was $100 \%$, ranging from 3 months to 11 years.

Twenty children had long-segment disease, and 18 of them underwent a definitive pull-through procedure. A higher proportion of these patients $(65 \%)$ presented in the newborn period than those with standard disease. One of the 20 had aganglionosis to the descending colon, 5 to the splenic flexure, 1 to the midtransverse colon, and 3 to the hepatic flexure. In 6 children, the disease involved the entire colon; one child had aganglionosis to the distal jejunum and another to the proximal jejunum. Definitive therapy was not accomplished in the remaining 2 infants because of near-total intestinal aganglionosis in 1 and death due to associated severe congenital anomalies 2 months following ileostomy in the other. There were equal numbers of males and females in the group with longsegment disease. The follow-up was complete, ranging from 4 months to 9 years.

Of the remaining 24 patients, 12 were referred for management of complications following their original procedure (Table 2) and 12 for consideration of revision or repeat of the previous pull-through (Table 3 ). Among those who required treatment of complications not resulting in a repeat pullthrough procedure were 6 patients who had undergone a Swenson procedure and 4 who had undergone ERPT. The remaining 2 children had not yet had a definitive procedure; 1 was referred for nutritional management and the other required colostomy revision. The last group of 12 patients included 10 who had previously undergone a definitive proce- 
dure and required a second pull-through. The original operation was the Swenson procedure in 5 and the Duhamel in 5. Of the first 5 patients, 1 suffered from constipation, 1 had anastomotic disruption, and 3 had anastomotic strictures. Of those who had had a Duhamel pull-through, 4 had constipation and 1 had a stricture with abscess formation. One of the last 2 patients had previously undergone resection of ganglionated colon only and, in the other, aganglionic bowel was erroneously included in the original ERPT.

The diagnostic work-up and preoperative, operative, and postoperative care have been outlined in previous reports [2, 4]. The cornerstone of preoperative diagnosis is the suction rectal biopsy [1]. We have had no false-negative or false-positive reports with the use of this technique, but have not hesitated to perform full-thickness biopsies in those few cases where definitive diagnosis was not possible [1]. The leveling colostomy with frozen section confirmation of the presence of ganglion cells is the initial operative procedure. The attainment of 1 year of age or a weight of $20 \mathrm{lbs} .(9-10 \mathrm{k})$ is recommended as a general guideline for performance of the definitive procedure in those patients diagnosed as newborns or infants. We have found, with increasing experience, that it is often possible to operate as early as 7 to 8 months of age. In older patients, the interval between colostomy or ileostomy and definitive procedure is individualized, with the pull-through done as the initial and only procedure in a few selected cases. At admission for definitive ERPT, standard preoperative bowel preparation is carried out with a 48 -h clear liquid diet and $1 \%$ neomycin irrigations, then oral neomycin and erythromycin base during the $24 \mathrm{~h}$ immediately preceding surgery. We contine to use broadspectrum parenteral antibiotics in the perioperative period.

The operative technique, well detailed in prior publications $[1,2]$, uses a combined abdominal-perineal approach. At the completion of surgery, a silastic sump drain is left within the muscular cuff along with a Foley catheter for $24 \mathrm{~h}$ postoperatively. Feedings are begun within $48 \mathrm{~h}$, with the patient usually discharged on the 7 th postoperative day following rectal examination. Neither back-up ileostomy nor colostomy is routinely left in place.

\section{Results}

There have been no operative or postoperative deaths. Of the three patients that died, one had untreatable disease with aganglionosis extending to within $20 \mathrm{~cm}$ of the ligament of Treitz. This patient died of severe malnutrition 3 months following diagnosis. The second child, with standard rectosigmoid disease, died from the complications of congenital heart disease (endocardial cushion defect) 1.5 months after an uncomplicated ERPT. The third patient, who had aganglionosis to the mid-small bowel and short-bowel syndrome, suffered from the multiple congenital anomalies of the Smith-Lemli-Opitz syndrome and expired 2 months after ileostomy.

All 55 patients with standard rectosigmoid disease who have undergone ERPT and are 3 years of age or older are continent of stool. Postoperative enterocolitis, which was demonstrated clinically in 8 of these patients (15\%), has been self-limited in 5 . The remaining 3 ultimately required internal sphincterotomy, which successfully resolved the problem. Exploratory laparotomy and enterolysis for acute adhesive bowel obstruction was required in 3 patients, and 2 developed anal stenosis, both responding to dilatations with 1 requiring a temporary colostomy. There were $6 \mathrm{pa}$ tients with minor complications, including a stitch abscess and minor wound infections. One patient required reexploration for postoperative bleeding and was subsequently found to be factor VIII deficient. A temporary enema program was used in 5 children who had infrequent fecal soiling in the early postoperative period [7]. The major complication rate of $3.6 \%$ (2 patients) represents an anastomotic leak with peritonitis in 1 child and a cuff abscess in a second, each requiring temporary colostomy. These colostomies have since been closed, and the patients subsequently had excellent results.

Of the 20 patients with long-segment disease, 18 underwent ERPT as a definitive procedure. Follow-up was possible in $100 \%$ of these children as well, and ranged from 4 months to 9 years. There have been 2 minor wound infections, 1 incisional hernia which was repaired, and 3 single episodes of enterocolitis (16\%) which resolved spontaneously. An incorrect intraoperative pathologic diagnosis in the first child operated on in this series of 99 patients resulted in ERPT being done with aganglionic bowel. A successful Swenson pull-through was subsequently performed following the correct diagnosis of total colonic aganglionosis. All children in this group who have reached 3 years of age are continent and have, on the average, 3 to 4 stools per day.

Twelve patients, 7 of whom had standard rectosigmoid disease and 5 with long-segment disease, were referred to us following pull-through procedures at other institutions for treatment of complications which did not require revision or repeat pull-through. Six had previously undergone Swenson procedures. A rectovesical fistula in 1 of the 6 was successfully repaired. One child had a rectal stricture and required a permanent ileostomy, and 4 were successfully treated with an enema program, 2 for fecal incontinence and 2 for constipation. Four children were referred following en ERPT procedure. Colostomy was performed in 1 patient who had a cuff abscess with $180^{\circ}$ torsion of the pulled-through colon and was then closed following revision of the malpositioned bowel. This patient continues to show an excellent result, having 1-2 bowel movements per day. One patient presented with a cuff abscess 8 months following ERPT and required operative 
drainage and ureterolysis. Permanent colostomy was required in a patient with a severe stricture. The last of these 4 children had multiple episodes of entercolitis successfully managed with rectal dilatation and irrrigation. One of the last two patients was referred with severe nutritional depletion following ileostomy for total colonic aganglionosis and was successfully managed with parenteral nutrition, and 1 required revision of a leveling colostomy for a severe stricture.

Twelve patients, 11 with rectosigmoid aganglionosis and 1 with total colonic aganglionosis, were referred to our institution for consideration of reperformance of unsuccessful pull-through procedures. Five of these patients had undergone the Swenson procedure, 5 the Duhamel procedure, 1 ERPT, and 1 colectomy of ganglionated bowel. In the child who had had an ERPT, aganglionic bowel was used. He was managed by repeat ERPT, with an excellent result. The patient who had undergone excision of dilated ganglionated bowel at age 16 had an ERPT at age 54. He developed a rectal stricture postoperatively and has been successfully managed with periodic dilatations. Of the 5 patients originally treated with the Swenson procedure, four have been reoperated upon with the same procedure and one with unmanageable constipation has undergone rectal myotomy alone, with excellent results. Three of 4 redos were done for severe rectal stricture and the fourth for anastomotic disruption. Of the 5 patients who previously underwent the Duhamel procedure, 3 presented with constipation secondary to a retained septum and septectomy was curative. In the fourth, the Duhamel procedure was redone for a severe rectal stricture. In the fifth, repeat pull-through was attempted but a satisfactory reconstruction was impossible, and the patient now has a permanent colostomy.

\section{Discussion}

As experience with and follow-up of the modified endorectal pull-through procedure increase, the advantages of this operation are underscored. Swenson first presented the abdominal-perineal resection and pull-through for the treatment of Hirschsprung's disease in 1948 [7], and his excellent results with the procedure stimulated its wider application. Comparable results were not realized by others, however, mainly because of the technical difficulties involved. This operation, in inexperienced hands, carries with it an increased risk of damage to the sacral nerves and presacral plexus responsible for bowel, bladder, and sexual function. Although technically easier to perform, the Duhamel retrorectal pull-through, introduced in 1956, has been demonstrated over time to have an increased incidence of incontinence, fecalomas in the retained rectum, and a retained septum in cases in which it has been improperly performed. The ERPT, first introduced by Soave [8] in 1964, with its dissection done entirely within the rectal wall, is technically easier than the Swenson procedure and has the advantage of avoiding damage to the sacral nerves. Continued experience with modifications of the ERPT by Boley [1] and Corar and Weintraub [2] has resulted in realization of these theoretical advantages.

The report of the survey of the Surgical Section of the American Academy of Pediatrics (AAP) on Hirschsprung's disease by Kleinhaus et al. [6] in 1979 is one of several collected series which can be used for a critical comparison of the various procedures (Table 4). Among patients with standard rectosigmoid disease, mortality in this large collected series was $1.1 \%$ in patients undergoing ERPT, 2.5\% in those having the Swenson procedure, and $1.8 \%$ in those managed by the Duhamel procedure. In comparison, in a 25-year follow-up study [10], Swenson et al. reported a $3.3 \%$ mortality in 483 of his own patients. Mortality in our series is $0 \%$, and even with the inclusion of our patient who died of complications of severe cardiac disease, would be only $1.4 \%$. Furthermore, if we consider the patients with total colonic Hirschsprung's disease separately, our mortali-

Table 4. Comparison of the various pull-through procedures in Hirschsprung's disease

\begin{tabular}{|c|c|c|c|c|c|}
\hline Series & $\begin{array}{l}\text { Standard } \\
\text { disease } \\
\text { mortality }\end{array}$ & $\begin{array}{l}\text { Long-segment } \\
\text { disease } \\
\text { mortality }\end{array}$ & $\begin{array}{l}\text { Permanent } \\
\text { enterostomy }\end{array}$ & $\begin{array}{l}\text { Postoperative } \\
\text { enterocolitis }\end{array}$ & $\begin{array}{l}\text { Percentage } \\
\text { without } \\
\text { complication }\end{array}$ \\
\hline Swenson (1975) [10] & $3.3 \%$ & & $4 \%$ & $16 \%$ & \\
\hline $\begin{array}{l}\text { Kleinhaus et al. (197 } \\
\text { ERPT } \\
\text { Swenson } \\
\text { Duhamel }\end{array}$ & $\begin{array}{l}1.1 \% \\
2.5 \% \\
1.8 \%\end{array}$ & $\begin{array}{r}0 \% \\
30 \% \\
25 \%\end{array}$ & $\begin{array}{l}1.1 \% \\
3.2 \% \\
1.1 \%\end{array}$ & $\begin{array}{r}2.1 \% \\
15.6 \% \\
5.9 \%\end{array}$ & $\begin{array}{l}60 \% \\
52 \% \\
55 \%\end{array}$ \\
\hline Polley et al. (1985) & $0 \%$ & $0 \%$ & $0 \%$ & $15 \%$ & $85 \%$ \\
\hline
\end{tabular}


ty is $0 \%$ compared to $0 \%$ in patients undergoing the ERPT, 30\% in those with the Swenson procedure, and $25 \%$ in those treated with the Duhamel procedure as reported in the AAP series [6].

An additional and very important parameter that can be used to compare the three procedures is the continence rate as reflected by the incidence of postoperative permanent enterostomy. None of the patients who were primarily diagnosed and treated at our institution has a permanent colostomy or ileostomy, and all patients who have reached the age of 3 years, whether with standard rectosigmoid disease or long-segment disease, are continent. In Swenson's own series, 4\% of the patients required permanent enterostomy, as did $3.2 \%$ of patients with Swenson procdures in the AAP Survey. The same collected series included a $1.1 \%$ incidence of permanent enterostomy for patients undergoing ERPT of the Duhamel procedure.

Our incidence of postoperative enterocolitis compares quite favorably with the other series. Swenson documents a postoperative enterocolitis rate of $16 \%$, while the AAP survey reported a $15.6 \%$ incidence after the Swenson procedure, $5.9 \%$ following the Duhamel procedure and 2.1\% following ERPT. In comparison, the postoperative enterocolitis rate in our series was $15 \%$. Perhaps most important is the overall morbidity: $85 \%$ of the patients in our series with standard rectosigmoid disease and $83 \%$ of those with long-segment aganglionosis had no complications, as compared to the American Academy of Pediatrics series of $52 \%$ with the Swenson procedure, 55\% with the Duhamel procedure, and $60 \%$ after ERPT.

The last several years have seen a much broader application of ERPT to the adult population, specifically in those patients with familial polyposis and ulcerative colitis [3]. The modifications introduced most recently have increased the technical ease of both endorectal dissection and anastomosis, as reflected in the low complication rate. As the procedure gains increased popularity, the ease with which it can be taught to surgical residents assumes even greater importance. At our institution, we have found that the results obtained after ERPT in children and adults with ulcerative colitis and familial polyposis compare quite favorably with those seen in our series of patients with Hirschsprung's disease.

Our approach to the diagnosis and treatment of infants and children with supected Hirschsprung's disease continues to utilize suction rectal biopsy for diagnosis, initial leveling enterostomy, and ERPT as the definitive procedure. Continued experience has led us to perform the definitive procedure for Hirschsprung's disease earlier in life. This experience with consistent use of the ERPT procedure over the past 11 years supports its continued application in patients with all forms of aganglionosis.

\section{References}

1. Boley SJ (1964) New modification of the surgical treatment of Hirschsprung's disease. Surgery 56: 1015-1017

2. Coran AG, Weintraub WH (1976) Modification of the endorectal procedure for Hirschsprung's disease. Surg Gyn Obstet 143: 1-6

3. Coran AG, Sarahan TM, Dent TL, Fiddian-Green R, Wesley JR, Jordan FT (1983) The endorectal pull-through for the management of ulcerative colitis in children and adults. Ann Surg 197: 99-105

4. Jordan FT, Coran AG, Weintraub WH, Wesley JR (1979) An evaluation of the modified endorectal procedure for Hirschsprung's disease. J Pediatr Surg 14: 681-685

5. Klein MD, Coran AG, Wesley JR, Drongowski RA (1984) Hirschsprung's disease in the newborn. J Pediatr Surg 19: 370-374

6. Kleinhaus S, Boley SJ, Sheran M, Sieber WK (1979) Hirschsprung's disease, a survey of the members of the Surgical Section of the American Academy of Pediatrics. J Pediatr Surg 14: 588-597

7. Sarahan T, Weintraub WH, Coran AG, Wesley JR (1982) The successful management of chronic constipation in infants and children. J Pediatr Surg 17: 171-174

8. Soave F (1964) Hirschsprung's disease: a new surgical technique. Arch Dis Child 39: 116

9. Swenson O, Nieuhauser BD, Pickett LK (1949) New concepts of the etiology, diagnosis and treatment of congenital megacolon (Hirschsprung's disease). Pediatrics 4: 201-209

10. Swenson O, Sherman JO, Fisher JH, Cohen E (1975) The treatment and postoperative complications of congenital megacolon: a 25 year follow-up. Ann Surg 182: 266-273

11. Weintraub WH, Heidelberger KP, Coran AG (1977) A simplified approach to diagnostic rectal biopsy in infants and children. Am J Surg 134: 307-310 